\title{
Quantum Thermodynamics of Nonequilibrium. Onsager Reciprocity and Dispersion-Dissipation Relations
}

\author{
Gian Paolo Beretta ${ }^{1}$ \\ Received March 13, 1984; revised September 30, 1986
}

\begin{abstract}
A generalized Onsager reciprocity theorem emerges as an exact consequence of the structure of the nonlinear equation of motion of quantum thermodynamics and is valid for all the dissipative nonequilibrium states, close and far from stable thermodynamic equilibrium, of an isolated system composed of a single constituent of matter with a finite-dimensional Hilbert space. In addition, a dispersiondissipation theorem results in a precise relation between the generalized dissipative conductivity that describes the mutual interrelation between dissipative rates of a pair of observables and the codispersions of the same observables and the generators of the motion. These results are presented together with a review of quantum thermodynamic postulates and general results.
\end{abstract}

\section{INTRODUCTION}

The purpose of this paper is to present a rigorous proof and generalization of Onsager's theory of irreversible processes, based uniquely and without additional assumptions or approximations on the nonlinear equation of motion recently proposed by the present author as the dynamical principle of quantum thermodynamics. ${ }^{(1)}$

The paper will be restricted to the quantum thermodynamics of an isolated system composed of a single constituent of matter with finitedimensional Hilbert space. However, we conjecture that with suitable technical refinements it can be extended to the general infinite-dimensional case, as well as to a general composite system. ${ }^{(2)}$

\footnotetext{
' Massachusetts Institute of Technology, Cambridge, Massachusetts 02139, and Politecnico di Milano, Milano, Italy.
} 
In 1931, Onsager published two papers ${ }^{(3)}$ in which he unified a large body of existing empirical observations on the nature of irreversible processes by formulating a general phenomenological theory based on his famous relations expressing the reciprocity of the mutual interrelations between different irreversible rate processes simultaneously occurring in a single system.

Onsager's reciprocal relations "appear to be universally valid although they cannot be proved by thermodynamics or by considerations on macroscopic symmetry." "(4) These relations cannot be derived in any rigorous way from the traditional Hamiltonian dynamical principles, i.e., from the Hamilton and the Liouville equations of classical dynamics or from the Schrödinger and the von Neumann equations of quantum dynamics. Indeed, as is well known, these equations can describe reversible processes only and, therefore, it is impossible to infer from them even the very existence of irreversible processes. This fundamental difficulty is often referred to as the irreversibility paradox. ${ }^{(5)}$

The irreversibility paradox notwithstanding, important theoretical arguments in support of the fundamental validity of Onsager's reciprocal relations have been given by Onsager, ${ }^{(3)}$ Casimir, ${ }^{(4)} \mathrm{Callen},{ }^{(6)} \mathrm{Kubo},{ }^{(7)}$ and others. ${ }^{(8-12)}$ In different ways, they have shown that the reciprocal relations can only be derived by complementing the known dynamical principles with some reasonable additional principle (e.g., the so-called "principle of microscopic reversibility"), assumption (e.g., that of sufficiently small perturbations, to ensure a linear behavior of the system), or approximation (e.g., that of sufficiently small deviations from thermodynamic equilibrium). Levine $^{(13)}$ has also shown that the reciprocal relations can be derived and extended to a higher nonequilibrium domain by adopting the assumption (or approximation) that systems proceed along a path continuously maximizing the entropy functional subject to some (possibly unknown) set of linear time-dependent constraints.

Recently, Hatsopoulos and Gyftopoulos ${ }^{(14)}$ have argued that the irreversibility paradox would be resolved if we realized that traditional Hamiltonian mechanics, from which the unitary dynamical postulate of orthodox quantum theory evolved, is incomplete, in the sense that it describes successfully only a limited aspect of microscopic physical reality. They proposed a broader quantum kinematics which contains orthodox quantum kinematics as a special case, and thermodynamics of stable equilibrium as another special case.

Thus, mechanics and thermodynamics can be unified into one uncontradictory science. The gap between mechanics and thermodynamics can be bridged without resorting to any of the usual statistical, phenomenological, or information-theoretic reasoning. The hardly definable notion of 
macroscopic versus microscopic reality plays no fundamental role. In the hierarchy of physical laws, the second law of thermodynamics is raised to the same level as the fundamental laws of mechanics, such as the great conservation principles. Entropy emerges as an intrinsic property of each of the microscopic constituents of material systems, much in the same way as energy is universally understood to be an intrinsic property of matter.

Hatsopoulos and Gyftopoulos concluded that traditional Hamiltonian dynamics is valid when restricted to the domain of orthodox quantum kinematics, but in the broader domain of their unified theory must be replaced with a new dynamical principle, encompassing only as a special case the familiar unitary evolutions of traditional Hamiltonian mechanics.

The present author ${ }^{(1,2,15,16)}$ has proposed a novel nonlinear equation of motion which, in the domain of orthodox kinematics, satisfies all the very restrictive requirements imposed by mechanics and, in the broader domain of the Hatsopoulos-Gyftopoulos kinematics, satisfies all of the very restrictive requirements imposed by general thermodynamics. The new quantum theory that emerges by adopting the Hatsopoulos-Gyftopoulos kinematics and the new equation of motion as the dynamical principle is called quantum thermodynamics.

We adopted the new principle of motion because it has the following general features. It is satisfied by all the unitary evolutions of mechanical states generated by the Schrödinger equation of motion and, therefore, when restricted to the orthodox mechanical states, quantum thermodynamics reduces to quantum mechanics. It causes, in general, nonunitary irreversible evolutions of the nonequilibrium states that are contemplated within quantum thermodynamics but not within quantum mechanics. It preserves the mean values of the energy and the other invariants of an isolated system, but it causes the value of the entropy to increase until the state reaches stable equilibrium. For each set of mean values of the energy and the other invariants, the equation of motion of quantum thermodynamics admits many equilibrium states, but among them one and only one is stable. Hence, the second law of thermodynamics ${ }^{(14)}$ emerges as a theorem of the new microscopic dynamical principle.

The irreversibility paradox is resolved within quantum thermodynamics because the new equation of motion, depending on the initial state of the system, can describe both reversible and irreversible processes. In this paper, we show that it is the structure of the non-Hamiltonian part of the equation of motion which entails a general theorem on the reciprocity of mutual interrelations between simultaneous dissipative rates of different observables.

Our Onsager reciprocity theorem offers a new perspective on the 
microscopic nature of irreversible processes, and follows from our dynamical principle with no need for additional principles, assumptions, or approximations. Consistently with the fact that they describe an important feature of irreversibility, the Onsager relations emerge in our theory from the terms in the equation of motion that are responsible for irreversibility and not, as in all the known derivations, from the irreducibly reversible Hamiltonian terms that can only describe unitary reversible evolution.

Another important feature of irreversibility was recognized by Callen $^{(6,9,17)}$ in his generalization of Nyquist's relation ${ }^{(18)}$ between voltage "fluctuations" or "noise" in a resistor at thermodynamic equilibrium and the electric conductivity of the resistor. Callen's theory, often referred to as the fluctuation-dissipation "theorem," expresses a direct relation between the generalized conductivity expressing the mutual interrelation between simultaneous dissipative rates of any pair of observables, and the covariance of measurement results, that we will call codispersion, of the same pair of observables.

As pointed out explicitly by Onsager and Machlup,${ }^{(10)}$ also the dispersion-dissipation "theorem" cannot be derived in any rigorous way from the traditional Hamiltonian dynamical principles, unless these are complemented by an additional postulate closely related to the additional principles, assumptions, or approximations needed to "derive" Onsager's reciprocity "theorem." Again, this is just another aspect of the irreversibility paradox, namely, of the impossibility to infer any feature of irreversibility (including its very existence) from the irreducibly reversible dynamical principle of orthodox unitary Hamiltonian mechanics. Again, our derivation based on quantum thermodynamics and its nonlinear, generally non-Hamiltonian equation of motion, not only extends such relations to all nonequilibrium states, not necessarily close to thermodynamic equilibrium, but also resolves the main conceptual difficulty of the traditional derivations, ${ }^{(3,6,7)}$ namely, the traditional paradoxical conclusion that the essential features of irreversibility follow from the fundamentally reversible Hamiltonian dynamics and the additional principle of "microscopic reversibility."

The postulates of quantum thermodynamics are reviewed briefly in Sec. 2. Our results on irreversibility are proved in Sec. 3 .

\section{POSTULATES OF QUANTUM THERMODYNAMICS}

Four postulates of quantum thermodynamics that define the Hatsopoulos-Gyftopoulos kinematics are as follows. ${ }^{(1,14)}$ 


\section{Postulate 1: Systems}

To every physical system there corresponds a complex, separable, complete inner produce space, a Hilbert space $\mathscr{H}$.

The Hilbert space $\mathscr{H}$ is the same space that orthodox quantum theory associates with the system. For simplicity, throughout this paper $\mathscr{H}$ is assumed to be finite dimensional. We denote by $\mathscr{L}(\mathscr{H})$ the set of all linear operators on $\mathscr{H}$. If equipped with the real scalar product defined by $(A, B)=\frac{1}{2} \operatorname{Tr}\left(A^{\dagger} B+B^{\dagger} A\right)$, where $\operatorname{Tr}$ denotes the trace functional on $\mathscr{H}$ and $A^{\dagger}$ the adjoint of operator $A$, then $\mathscr{L}(\mathscr{H})$ becomes a Hilbert space on the real scalars. We denote by $\mathscr{L}_{s}(\mathscr{H})$ the subspace of $\mathscr{L}(\mathscr{H})$ of all self-adjoint linear operators on $\mathscr{H}$. Also $\mathscr{L}_{s}(\mathscr{H})$, with the real scalar product just defined, is a Hilbert space. Finally, we denote by $\mathscr{P}(\mathscr{H})$ the subset of $\mathscr{L}_{s}(\mathscr{H})$ of all the unit-trace, nonnegative-definite, self-adjoint linear operators on $\mathscr{H}$.

\section{Postulate 2: Correspondence Principle}

Some continuous linear real functionals $a(\cdot), b(\cdot), \ldots$ on $\mathscr{L}_{s}(\mathscr{H})$ correspond to physical observables of the system.

By the Riesz representation theorem, any continuous linear functional $a(\cdot)$ on the Hilbert space $\mathscr{L}_{s}(\mathscr{H})$ is generated by a unique element $A$ in $\mathscr{L}_{s}(\mathscr{H})$, such that

$$
a(X)=(A, X)=\operatorname{Tr} A X
$$

for every $X$ in $\mathscr{L}_{s}(\mathscr{H})$. Quantum thermodynamics does not exclude the existence of physical observables that are not represented by continuous linear functionals on $\mathscr{L}_{s}(\mathscr{H})$. For example, entropy is represented by a nonlinear functional defined on the subset $\mathscr{P}(\mathscr{H})$ of $\mathscr{L}_{s}(\mathscr{H})$.

\section{Postulate 3: States}

To every state of a physical system, there corresponds a unit-trace, nonnegative-definite, self-adjoint linear operator $\rho$ on $\mathscr{H}$, i.e., an element of $\mathscr{P}(\mathscr{H})$. Operator $\rho$ is called the state operator.

The state operators that are idempotent, i.e., such that $\rho^{2}=\rho$, represent the states of orthodox quantum kinematics. They are all contained within the broader kinematics implied by Postulate 3 . 


\section{Postulate 4: Value of a Physical Observable}

The value of a physical observable represented by a (linear or nonlinear) real functional $a(\cdot)$, defined on $\mathscr{L}_{s}(\mathscr{H})$ or at least on $\mathscr{P}(\mathscr{H})$, for a system in a state represented by state operator $\rho$ is given by the value $a(\rho)$.

For a linear $a(\cdot)$, generated by the self-adjoint linear operator $A$ on $\mathscr{H}$, Eq. (1) yields $a(\rho)=\operatorname{Tr} A \rho$. For the nonlinear functional representing the entropy, ${ }^{(1,14)}$ the value corresponding to state $\rho$ is given by $s(\rho)=-k_{\mathrm{B}} \operatorname{Tr} \rho \ln \rho$, where $k_{\mathrm{B}}$ is the Boltzmann constant.

The dynamical postulate of quantum thermodynamics depends on the internal structure of the system. ${ }^{(2)}$ In this paper, we consider only systems composed of a single constituent such as a single material particle or a field. Each constituent is characterized by a set of generators of the motion $^{(1)}$ always including the identity operator $I$ and the Hamiltonian operator $H$, where $H$ is the same Hamiltonian operator that orthodox quantum theory associates with the constituent. However, constituents such as Bose-Einstein and Fermi-Dirac fields, for which $\mathscr{H}$ is a Fock space, have other generators of the motion in addition to $I$ and $H$, i.e., the number operators $N_{1}, \ldots, N_{n}$ for each of the $n$ types of material particles in the field.

\section{Postulate 5: Equation of Motion for a Single Constituent}

For a system consisting of a single constituent, i.e., a single particle or a Bose-Einstein or Fermi-Dirac field, the state operator $\rho$ evolves according to the equation

$$
\frac{d \rho}{d t}=-\frac{i}{\hbar}[H, \rho]-\frac{1}{\tau} F(\rho)
$$

where $[H, \rho]=H \rho-\rho H$, and $F(\rho)$ is a linear, self-adjoint operator on $\mathscr{H}$ defined as a nonlinear function of the state operator $\rho$ by

$$
\begin{aligned}
& F(\rho)=\frac{1}{2}\left[\sqrt{\rho} D(\rho)+D^{\dagger}(\rho) \sqrt{\rho}\right] \\
& D(\rho)=\sqrt{\rho} \ln \rho-(\sqrt{\rho} \ln \rho)_{L\left(\sqrt{\rho} \cdot \sqrt{\rho} H, \sqrt{\rho} N_{1}, \ldots, \sqrt{\rho} N_{n}\right)}
\end{aligned}
$$

where $\tau$ is an internal-dissipation characteristic time of the constituent, $L\left(\sqrt{\rho}, \sqrt{\rho} H, \sqrt{\rho} N_{1}, \ldots, \sqrt{\rho} N_{n}\right)$ denotes the linear manifold in $\mathscr{L}(\mathscr{H})$ spanned by all linear combinations with real coefficients of operators $\sqrt{\rho}$, $\sqrt{\rho} H, \sqrt{\rho} N_{1}, \ldots, \sqrt{\rho} N_{n}$, and $(\sqrt{\rho} \ln \rho)_{L}$ denotes the orthogonal projection within $\mathscr{L}(\mathscr{H})$ of $\sqrt{\rho}$ onto the linear manifold $L$. Operator $I$ is the identity on $\mathscr{H}$ and $H$ the Hamiltonian of the constituent. Operators $N_{1}, \ldots, N_{n}$ all commute with $H$ and are called the non-Hamiltonian 
generators of the motion of the constituent. The number $n$ of nonHamiltonian generators depends on the constituent and may be zero for some constituents.

The internal-dissipation characteristic time $\tau$ in Eq. (2) cannot be inferred other than from experiments on the relaxation of nonequilibrium states. Mathematically, all the general results that we summarize and derive below unfold identically whether $\tau$ is a universal constant, a constant that depends on the type of constituent, or any positive functional of $\rho$. At present, the problem of estimating $\tau$ on the basis of available experimental data remains unresolved. However, we have discussed specific nontrivial implications of Eq. (2) which should in principle lead to experimental verification. ${ }^{(15)}$

Geometrically, the operator $F(\rho)$ can be visualized as the projection of the gradient of the entropy functional $-k_{\mathrm{B}} \operatorname{Tr} \rho \ln \rho$ onto the hyperplane generated by the normalization functional $\operatorname{Tr} \rho$, the energy functional $\operatorname{Tr} H \rho$, and (for a field) each of the number-of-particle functionals $\operatorname{Tr} N_{1} \rho, \ldots, \operatorname{Tr} N_{n} \rho$. The two terms in Eq. (2) compete with each other in the sense that $-i[H, \rho] / \hbar$ tends to "pull" $\rho$ in a unitary motion tangent to the local constant entropy hypersurface whereas $-F(\rho) / \tau$ tends to "pull" $\rho$ in the local direction of steepest entropy ascent while maintaining it on a constant energy and constant number of particles hyperplane.

The unitary Hamiltonian term $-i[H, \rho] / h$ maintains invariant the entropy functional by maintaining invariant each of the eigenvalues of the state operator $\rho$. If $H$ is time dependent, then the equation of motion describes an adiabatic exchange of energy between the system and other external systems during which the system remains uncorrelated. The adiabatic rate of energy exchange, $\operatorname{Tr}(d H / d t) \rho$, depends on the rate of change of the Hamiltonian operator $H$. The nonunitary internal-dissipation term $-F(\rho) / \tau$ does not contribute to changing the values of the energy functional and the number-of-particle functionals even if $H$ is time dependent. However, it causes an irreversible increase in the value of the entropy for all nonequilibrium states not belonging to a limit cycle. Interestingly, for processes described by a time-dependent $H$, the rate of entropy production does not depend on the rate of change $d H / d t$ of the Hamiltonian and, thus, we conclude that in the limit of very fast changes in $H$ we can achieve adiabatic exchanges of energy with negligible internal production of entropy, because the faster is the energy exchange the shorter is the time available for the internal-dissipation term to generate entropy.

The magnitude of the rate of entropy production [see Eq. (24) below] is a nonlinear function of $\rho$ which goes to zero smoothly at many states, including the idempotent states of quantum mechanics, the equilibrium states, and the limit cycles. It is therefore interesting to note that if a state is 
very close to, say, an idempotemt state, then the term $-F(\rho) / \tau$ may be so small as compared to the term $-i[H, \rho] / \hbar$ that its effect may be relatively negligible for a long time, during which the evolution may seem dominated by the unitary term $-i[H, \rho] / \hbar$. According to Eq. (2), however, all the idempotent states, the limit cycles, and the less-than-maximum-entropy equilibrium states are unstable in the sense of Lyapunov, i.e., arbitrarily close to each one of them there is a trajectory that after some finite time (perhaps very long) carries the state to a finite distance. The only equilibrium states that are stable in the sense of Lyapunov are the maximum entropy states, ${ }^{(19)}$ i.e., the equilibrium states of classical thermodynamics.

Given an operator $V$ and a linear manifold $L$ in $\mathscr{L}(\mathscr{H})$, the projection $V_{L}$ of $V$ onto $L$ is the unique element in $L$ such that $\left(V_{L}, X\right)=(V, X)$ for every $X$ in $L$. The theory of Gram determinants offers a useful explicit way to write ${ }^{(16)} V_{L}$ and, in particular, the orthogonal projection of $\sqrt{\rho} \ln \rho$ onto $L\left(\sqrt{\rho}, \sqrt{\rho} H, \sqrt{\rho} N_{1}, \ldots, \sqrt{\rho} N_{n}\right)$ which is part of the definition of the "internal dissipation" term $-F(\rho) / \tau$ in the equation of motion. For each given state operator $\rho$, we denote by $R_{1}, \ldots, R_{z}$ a set of self-adjoint operators such that the operators $\sqrt{\rho}, \sqrt{\rho} R_{1}, \ldots, \sqrt{\rho} R_{z}$ span the linear manifold $L\left(\sqrt{\rho}, \sqrt{\rho} H, \sqrt{\rho} N_{1}, \ldots, \sqrt{\rho} N_{n}\right)$ and are linearly independent, i.e., such that the Gram determinant

$$
\left|\begin{array}{cccc}
\left(\sqrt{\rho} R_{0}, \sqrt{\rho} R_{0}\right) & \left(\sqrt{\rho} R_{0}, \sqrt{\rho} R_{1}\right) & \cdots & \left(\sqrt{\rho} R_{0}, \sqrt{\rho} R_{z}\right) \\
\left(\sqrt{\rho} R_{1}, \sqrt{\rho} R_{0}\right) & \left(\sqrt{\rho} R_{1}, \sqrt{\rho} R_{1}\right) & \cdots & \left(\sqrt{\rho} R_{1}, \sqrt{\rho} R_{z}\right) \\
\vdots & \vdots & \ddots & \vdots \\
\left(\sqrt{\rho} R_{z}, \sqrt{\rho} R_{0}\right) & \left(\sqrt{\rho} R_{z}, \sqrt{\rho} R_{1}\right) & \cdots & \left(\sqrt{\rho} R_{z}, \sqrt{\rho} R_{z}\right)
\end{array}\right|
$$

where $R_{0}$ denotes the identity operator $I$, is different from zero and, therefore, is strictly positive.

As a useful shorthand notation, given any two self-adjoint operators $A$ and $B$, we will denote $(\sqrt{\rho} A, \sqrt{\rho} B)$ by $\langle A B\rangle$, i.e.,

$$
\langle A B\rangle=(\sqrt{\rho} A, \sqrt{\rho} B)=\frac{1}{2} \operatorname{Tr} \rho\{A, B\}
$$

where the anticommutator $\{A, B\}=A B+B A$. We will denote the strictly positive Gram determinant in Eq. (5) by $G\left(\rho ; R_{0}, R_{1}, \ldots, R_{z}\right)$, i.e.,

$$
G\left(\rho ; R_{0}, R_{1}, \ldots, R_{z}\right)=\left|\begin{array}{cccc}
\left\langle R_{0} R_{0}\right\rangle & \left\langle R_{0} R_{1}\right\rangle & \cdots & \left\langle R_{0} R_{z}\right\rangle \\
\left\langle R_{1} R_{0}\right\rangle & \left\langle R_{1} R_{1}\right\rangle & \cdots & \left\langle R_{1} R_{z}\right\rangle \\
\vdots & \vdots & \ddots & \vdots \\
\left\langle R_{z} R_{0}\right\rangle & \left\langle R_{z} R_{1}\right\rangle & \cdots & \left\langle R_{z} R_{z}\right\rangle
\end{array}\right|
$$


In terms of this notation, the orthogonal projection of an operator $\sqrt{\rho} V$, where $V$ is self-adjoint, onto the linear manifold $L\left(\sqrt{\rho} I, \sqrt{\rho} H, \sqrt{\rho} N_{1}, \ldots, \sqrt{\rho} N_{n}\right)$ may be written as

$$
\begin{aligned}
& (\sqrt{\rho} V)_{L\left\{\sqrt{\rho}, \sqrt{\rho} H, \sqrt{\rho} N_{1}, \ldots \sqrt{\rho} N_{n}\right\}} \\
& =-\frac{\left|\begin{array}{ccccc}
0 & \sqrt{\rho} R_{0} & \sqrt{\rho} R_{1} & \cdots & \sqrt{\rho} R_{z} \\
\left\langle R_{0} V\right\rangle & \left\langle R_{0} R_{0}\right\rangle & \left\langle R_{0} R_{1}\right\rangle & \cdots & \left\langle R_{0} R_{z}\right\rangle \\
\left\langle R_{1} V\right\rangle & \left\langle R_{1} R_{0}\right\rangle & \left\langle R_{1} R_{1}\right\rangle & \cdots & \left\langle R_{1} R_{z}\right\rangle \\
\vdots & \vdots & \vdots & \ddots & \vdots \\
\left\langle R_{z} V\right\rangle & \left\langle R_{z} R_{0}\right\rangle & \left\langle R_{z} R_{1}\right\rangle & \cdots & \left\langle R_{z} R_{z}\right\rangle
\end{array}\right|}{G\left(\rho ; R_{0}, R_{1}, \ldots, R_{z}\right)}
\end{aligned}
$$

Using Eq. (8) with $\sqrt{\rho} V=\sqrt{\rho} \ln \rho$ and substituting in Eq. (5), the operator $F(\rho)$ in the equation of motion may also be written as

$$
F(\rho)=\frac{\left|\begin{array}{ccccc}
\rho \ln \rho & \frac{1}{2}\left\{\rho, R_{0}\right\} & \frac{1}{2}\left\{\rho, R_{1}\right\} & \cdots & \frac{1}{2}\left\{\rho, R_{z}\right\} \\
\left\langle R_{0} \ln \rho\right\rangle & \left\langle R_{0} R_{0}\right\rangle & \left\langle R_{0} R_{1}\right\rangle & \cdots & \left\langle R_{0} R_{z}\right\rangle \\
\left\langle R_{1} \ln \rho\right\rangle & \left\langle R_{1} R_{0}\right\rangle & \left\langle R_{1} R_{1}\right\rangle & \cdots & \left\langle R_{1} R_{z}\right\rangle \\
\vdots & \vdots & \vdots & \ddots & \vdots \\
\left\langle R_{z} \ln \rho\right\rangle & \left\langle R_{z} R_{0}\right\rangle & \left\langle R_{z} R_{1}\right\rangle & \cdots & \left\langle R_{z} R_{z}\right\rangle
\end{array}\right|}{G\left(\rho ; R_{0}, R_{1}, \ldots, R_{z}\right)}
$$

where $\left\langle R_{i} \ln \rho\right\rangle=\left(\sqrt{\rho} R_{i}, \sqrt{\rho} \ln \rho\right)=\operatorname{Tr} R_{i} \rho \ln \rho$.

The operator $F(\rho)$ has many interesting features. ${ }^{(1,16)}$ It reduces to the null operator whenever $\rho^{2}=\rho$, namely, for each quantum mechanical state. Equation (2) maintains idempotent any initially idempotent state operator and, therefore, all the unitary evolutions of mechanical states generated by the Schrödinger equation are also solutions of Eq. (2), and we conclude that quantum thermodynamics contains the whole of quantum mechanics as a special case. But quantum thermodynamics is more general, because for the nonmechanical states, i.e., for $\rho^{2} \neq \rho, F(\rho)$ does indeed contribute nontrivially to the time evolution. In the next section we study the features of such contribution that are due to the structure of operator $F(\rho)$.

\section{QUANTUM THERMODYNAMICS OF NONEQUILIBRIUM}

In this section, we study the implications of the postulates of quantum thermodynamics on the behavior of a single constituent in a general non- 
equilibrium state. Other general theorems that are proved in Ref. 1 will not be repeated here.

First we develop a useful way to represent a general quantum thermodynamic state, namely, an expression valid for all states, mechanical and nonmechanical, equilibrium and nonequilibrium (close and far from stable equilibrium).

We denote by $\left\{X_{0}, X_{1}, X_{2}, \ldots, X_{j}, \ldots\right\}$ a set of self-adjoint linear operators spanning the real space $\mathscr{L}_{s}(\mathscr{H})$ of all the self-adjoint linear operators on $\mathscr{H}$. If the operators in $\left\{X_{j}\right\}$ are also linearly independent, then we call $\left\{X_{j}\right\}$ a quorum. ${ }^{(20)}$ In what follows, we assume to have chosen a set $\left\{X_{3}\right\}$ once and for all in such a way that $X_{0}$ is the identity, i.e., $X_{0}=I$. The $X_{j}$ 's need not be linearly independent.

In terms of the operators in set $\left\{X_{0}=I, X_{j}\right\}$, any state operator $\rho$ can be written as

$$
\rho=\frac{B \exp \left(-\sum_{j} f_{j} X_{j}\right)}{\operatorname{Tr} B \exp \left(-\Sigma_{j} f_{j} X_{j}\right)}
$$

where $\sum_{j}$ denotes the summation over $j$ from 1 to $\operatorname{dim}(\mathscr{H}), f_{j}$ are real scalars, and $B$ is an idempotent operator obtained from $\rho$ by substituting the nonzero eigenvalues of $\rho$ with unity. Indeed, given any state $\rho$, we can construct operator $B$ as indicated and an operator $P$ by substituting the zero eigenvalues of $\rho$ with unity. Then, $\rho=B P=P B$ by construction and $P$ is strictly positive. Therefore, operator $\ln P$ is a well-defined element of $\mathscr{L}_{s}(\mathscr{H})$ and, as such, can be written as a linear combination of the operators in the set $\left\{X_{J}\right\}$, i.e., we can find real scalars $f_{0}, f_{1}, \ldots, f_{j}, \ldots$ such that

$$
\ln P=-f_{0} I-\sum_{j} f_{j} X_{j}
$$

Thus, we find

$$
\rho=B P=B \exp \left(f_{0} I-\sum_{j} f_{j} X_{j}\right)
$$

and, because $\rho$ is unit-trace, we may solve for $f_{0}$ and obtain Eq. (10).

For example, if $\rho$ is a mechanical state, i.e., it is idempotent $\left(\rho^{2}=\rho\right)$, then $B$ is a one-dimensional projection operator, i.e., $B^{2}=B$ and $\operatorname{Tr} B=1$, $P=I$, each $f_{j}=0$, and $\rho=B$. Again, if $\rho$ is a nondissipative state, ${ }^{(1)}$ i.e., a state for which $F(\rho)=0$, then it can be written as

$$
\rho=\frac{B \exp \left(-\beta H-\sum_{i=1}^{n} v_{i} N_{i}\right)}{\operatorname{Tr} B \exp \left(-\beta H-\sum_{i=1}^{n} v_{i} N_{i}\right)}
$$

Thus, if the first $n+1$ elements in the set $\left\{X_{j}\right\}$ are chosen so that $X_{0}=I$, $X_{1}=H, X_{2}=N_{1}, \ldots, X_{n+1}=N_{n}$, then Eq. (10) yields a nondissipative state 
whenever $f_{j}=0$ for every $j>n+1$ (as long as $\left\{X_{j}\right\}$ spans $\mathscr{L}_{s}(\mathscr{H})$ ). If, in addition, operator $B=I$, then Eq. (13) represents a stable equilibrium state. $^{(1,19)}$

We emphasize that Eq. (10) involves no additional assumptions or approximations. This way of writing an arbitrary state operator would not hold in general if, for example, we assume that $B=I$ and the operators $\left\{X_{j}\right\}$ do not form a complete set in the sense we specified. For example, the mathematical work of Levine ${ }^{(13)}$ holds only for a nonequilibrium state for which (in our notation) $B=I$ and the operators $\left\{X_{j}\right\}$ form a restricted set that does not span $\mathscr{L}_{s}(\mathscr{H})$ and, therefore, Levine's assumption that a system initially in such a special nonequilibrium state proceeds at all times only through such states should be checked for consistency with the internal dynamics of the system, i.e. (in our theory), with Eq. (2).

Using Eq. (10), and the fact that $\sqrt{\rho} \ln B$ is the null operator, we find

$$
\sqrt{\rho} \ln \rho=-\sum_{j} f_{j} \sqrt{\rho} X_{j}-\sqrt{\rho} I \operatorname{Tr} B \exp \left(-\sum_{j} f_{j} X_{j}\right)
$$

Using the linearity of determinants with respect to each row and column, the linearity of the trace functional, and the fact that $R_{0}=I$, Eq. (9) may be rewritten as

$$
F(\rho)=-\sum_{j} f_{j} \frac{\left|\begin{array}{ccccc}
\frac{1}{2}\left\{\rho, X_{j}\right\} & \frac{1}{2}\left\{\rho, R_{0}\right\} & \frac{1}{2}\left\{\rho, R_{1}\right\} & \cdots & \frac{1}{2}\left\{\rho, R_{z}\right\} \\
\left\langle R_{0} X_{j}\right\rangle & \left\langle R_{0} R_{0}\right\rangle & \left\langle R_{0} R_{1}\right\rangle & \cdots & \left\langle R_{0} R_{z}\right\rangle \\
\left\langle R_{1} X_{j}\right\rangle & \left\langle R_{1} R_{0}\right\rangle & \left\langle R_{1} R_{1}\right\rangle & \cdots & \left\langle R_{1} R_{z}\right\rangle \\
\vdots & \vdots & \vdots & \ddots & \vdots \\
\left\langle R_{z} R_{0}\right\rangle & \left\langle R_{z} R_{1}\right\rangle & \cdots & \left\langle R_{z} R_{z}\right\rangle
\end{array}\right|}{G\left(\rho ; R_{0}, R_{1}, \ldots, R_{z}\right)}
$$

We may now define the dissipative rate of change of the value $a(\rho)=\operatorname{Tr} A \rho$ of an observable represented by a time-independent continuous linear functional on $\mathscr{L}_{s}(\mathscr{H})$ by

$$
\frac{D a}{D t}=-\operatorname{Tr} A F(\rho) / \tau=(A,-F(\rho) / \tau)
$$

Clearly, the actual rate of change is given by the sum of the Hamiltonian rate and the dissipative rate according to the equation

$$
\frac{d a(\rho)}{d t}=\frac{i}{h} \operatorname{Tr} \rho[H, A]+\frac{D a(\rho)}{D t}
$$

where we used Eq. (2) for $d \rho / d t$. 
If we denote by $x_{j}(\rho)$ the value at state $\rho$ of the linear observable represented by the self-adjoint operator $X_{j}$ in the complete set $\left\{X_{j}\right\}$, i.e., $x_{j}(\rho)=\left(X_{j}, \rho\right)=\operatorname{Tr} X_{j} \rho=\left\langle X_{j} R_{0}\right\rangle$, then the values of the dissipative rates of these observables are sufficient to determine the operator $F(\rho)$. In other words, the system of equations

$$
\left(X_{j},-F(\rho) / \tau\right)=\frac{D x_{j}(\rho)}{D t}
$$

can be solved for $-F(\rho) / \tau$. Hence, giving the values of all the dissipative rates $D x_{j}(\rho) / D t$ is equivalent to giving the internal-dissipation term $-F(\rho) / \tau$, i.e., the non-Hamiltonian contribution to the rate of change of the state operator $\rho$.

Using Eq. (15) and (18), we find

$$
\frac{D x_{j}(\rho)}{D t}=\sum_{i} f_{i} L_{i j}
$$

where

$$
L_{i j}=\frac{1}{\tau} \frac{\left|\begin{array}{ccccc}
\left\langle X_{i} X_{j}\right\rangle & \left\langle X_{i} R_{0}\right\rangle & \left\langle X_{i} R_{1}\right\rangle & \cdots & \left\langle X_{i} R_{z}\right\rangle \\
\left\langle R_{0} X_{j}\right\rangle & \left\langle R_{0} R_{0}\right\rangle & \left\langle R_{0} R_{1}\right\rangle & \cdots & \left\langle R_{0} R_{z}\right\rangle \\
\left\langle R_{1} X_{j}\right\rangle & \left\langle R_{1} R_{0}\right\rangle & \left\langle R_{1} R_{1}\right\rangle & \cdots & \left\langle R_{1} R_{z}\right\rangle \\
\vdots & \vdots & \vdots & & \vdots \\
\left\langle R_{z} X_{j}\right\rangle & \left\langle R_{z} R_{0}\right\rangle & \left\langle R_{z} R_{1}\right\rangle & \cdots & \left\langle R_{z} R_{z}\right\rangle
\end{array}\right|}{G\left(\rho ; R_{0}, R_{1}, \ldots, R_{z}\right)}
$$

Because determinants are invariant upon transposition and $\langle A B\rangle=\langle B A\rangle$ by definition, the state functionals $L_{i j}$ satisfy the reciprocity relations

$$
L_{i j}=L_{j i}
$$

To interpret the relations just proved, let us write the entropy functional using Eq. (14). We find

$$
\begin{aligned}
s(\rho) & =-k_{\mathrm{B}} \operatorname{Tr} \rho \ln \rho=-k_{\mathrm{B}}(\sqrt{\rho}, \sqrt{\rho} \ln \rho) \\
& =k_{\mathrm{B}} \sum_{j} f_{j} x_{j}(\rho)+k_{\mathrm{B}} \operatorname{Tr} B \exp \left(-\sum_{j} f_{j} X_{j}\right)
\end{aligned}
$$


We can readily show that

$$
\left.\frac{\partial s(\rho)}{\partial x_{i}(\rho)}\right|_{x_{j \neq i}(\rho)}=k_{\mathrm{B}} f_{i}
$$

and, therefore, $k_{\mathrm{B}} f_{i}$ may be interpreted as the affinity or generalized "force" representing the entropy change that corresponds to an independent change in the value of the linear observable $x_{i}(\cdot)$. Equation (19) implies the general existence of linear interrelations between the dissipative rates or generalized "dissipative fluxes," $D x_{i}(\rho) / D t$, and the affinities or generalized "forces," $k_{\mathrm{B}} f_{i}$. The Onsager reciprocity relation (21) implies that the effect of the $j$ th affinity onto the $i$ th dissipative rate is identical to the effect of the $i$ th affinity onto the $j$ th dissipative rate. This reciprocity theorem is valid in general, namely, for all the nonequilibrium dissipative states $\rho$, regardless of whether they are close or far from thermodynamic equilibrium. The coefficients $L_{i j}$ may be called generalized "dissipative conductivities." As seen by inspection of Eq. (20), they are nonlinear functionals of the state $\rho$.

We have shown in Ref. 1 that the rate of change of the entropy is given by

$$
\frac{d s(\rho)}{d t}=\frac{k_{\mathrm{B}}}{\tau}(D(\rho), D(\rho))
$$

where $D(\rho)$ is the operator defined in Eq. (4). Clearly, $d s(\rho) / d t$ is nonnegative. It is equal to zero only for nondissipative states, i.e., states represented by Eq. (13). With the help of Eq. (14), we can also show that

$$
\frac{d s(\rho)}{d t}=k_{\mathrm{B}} \sum_{i} \sum_{j} f_{i} f_{j} L_{i j}
$$

or, using Eq. (19),

$$
\frac{d s(\rho)}{d t}=k_{\mathrm{B}} \sum_{j} f_{j} \frac{D x_{j}(\rho)}{D t}
$$

With the help of Eq. (8), we can readily verify that an alternative way to write Eq. (20) is

$$
L_{i j}=\frac{1}{\tau}\left(\sqrt{\rho} X_{i}-\left(\sqrt{\rho} X_{i}\right)_{L}, \sqrt{\rho} X_{j}-\left(\sqrt{\rho} X_{j}\right)_{L}\right)
$$


where $\left(\sqrt{\rho} X_{i}\right)_{L}$ and $\left(\sqrt{\rho} X_{j}\right)_{L}$ denote, respectively, the orthogonal projections of $\sqrt{\rho} X_{i}$ and $\sqrt{\rho} X_{j}$ onto the linear manifold $L=L\left(\sqrt{\rho}, \sqrt{\rho} H, \sqrt{\rho} N_{1}, \ldots, \sqrt{\rho} N_{n}\right)$. By virtue of Eq. (27), the matrix

$$
\left[L_{l j}\right]=\left|\begin{array}{ccccc}
L_{11} & L_{12} & \cdots & L_{1} & \cdots \\
L_{21} & L_{22} & \cdots & L_{2 j} & \cdots \\
\vdots & \vdots & \ddots & \vdots & \\
L_{i 1} & L_{i 2} & \cdots & L_{i j} & \\
\vdots & \vdots & & & \ddots
\end{array}\right|
$$

is a Gram matrix and, as such, it is nonnegative and its determinant is nonnegative, i.e.,

$$
\left|L_{i j}\right| \geqslant 0
$$

The determinant $\left|L_{i j}\right|$ is strictly positive only if the operators $\left\{\sqrt{\rho} X_{j}-\left(\sqrt{\rho} X_{j}\right)_{L}\right\}$ are linearly independent. Only in that case may Eq. (19) be solved to yield

$$
f_{i}=\sum_{j} \frac{D x_{j}(\rho)}{D t}\left(L^{-1}\right)_{j i}
$$

and the rate of entropy generation may be written as

$$
\frac{d s(\rho)}{d t}=k_{\mathrm{B}} \sum_{i} \sum_{j} \frac{D x_{i}(\rho)}{D t} \frac{D x_{j}(\rho)}{D t}\left(L^{-1}\right)_{j t}
$$

Clearly, the structure of relations (19), (25), (26), (30), and (31) is identical to the well-known relations that form the basis of the Onsager phenomenological theory of irreversible processes.

Equation (27) also shows an important direct relation between the dissipative conductivity $L_{i j}$ and the scalar product of the two operators $\sqrt{\rho} X_{i}-\left(\sqrt{\rho} X_{i}\right)_{L}$ and $\sqrt{\rho} X_{j}-\left(\sqrt{\rho} X_{j}\right)_{L}$, each of which is orthogonal to the linear manifold $L$.

We now define the codispersion of measurement results of a pair of linear observables $a(\cdot)$ and $b(\cdot)$ with associated self-adjoint operators $A$ and $B$, respectively, by the relation

$$
\langle\Delta A \Delta B\rangle=\left|\begin{array}{cc}
\langle A B\rangle & \langle A I\rangle \\
\langle I B\rangle & \langle I I\rangle
\end{array}\right|
$$

where $I$ is the identity operator and $\langle A B\rangle$ is defined by Eq. (6). The 
following are alternative relations for the codispersion of observables $A$ and $B$,

$$
\begin{aligned}
\langle A A A B\rangle & =\frac{1}{2} \operatorname{Tr} \rho\{A, B\}-(\operatorname{Tr} A \rho)(\operatorname{Tr} B \rho) \\
& \left.=\left(\sqrt{\rho} A-(\sqrt{\rho} A)_{\sqrt{\rho}}, \sqrt{\rho} B-\sqrt{\rho} B\right)_{\sqrt{\rho}}\right)
\end{aligned}
$$

where $(\sqrt{\rho} A)_{\sqrt{\rho}}$ denotes the orthogonal projection of $\sqrt{\rho} A$ onto the linear span of $\sqrt{\rho}$, i.e., by virtue of Eq. (8), $(\sqrt{\rho} A)_{\sqrt{\rho}}=\sqrt{\rho}\langle A I\rangle=\sqrt{\rho} \operatorname{Tr} A \rho$.

Using well-known properties of determinants, we can readily show that Eq. (20) may be rewritten as

$$
L_{i j}=\frac{1}{\tau} \frac{\left|\begin{array}{cccc}
\left\langle\Delta X_{i} \Delta X_{j}\right\rangle & \left\langle\Delta X_{i} \Delta R_{1}\right\rangle & \cdots & \left\langle\Delta X_{i} \Delta R_{z}\right\rangle \\
\left\langle\Delta R_{1} \Delta X_{j}\right\rangle & \left\langle\Delta R_{1} \Delta R_{1}\right\rangle & \cdots & \left\langle\Delta R_{1} \Delta R_{z}\right\rangle \\
\vdots & \vdots & \ddots & \vdots \\
\left\langle\Delta R_{z} \Delta X_{j}\right\rangle & \left\langle\Delta R_{z} \Delta R_{1}\right\rangle & \cdots & \left\langle\Delta R_{z} \Delta R_{z}\right\rangle
\end{array}\right|}{\left|\begin{array}{ccc}
\left\langle\Delta R_{1} \Delta R_{1}\right\rangle & \cdots & \left\langle\Delta R_{1} \Delta R_{z}\right\rangle \\
\vdots & \ddots & \vdots \\
\left\langle\Delta R_{z} \Delta R_{1}\right\rangle & \cdots & \left\langle\Delta R_{z} \Delta R_{z}\right\rangle
\end{array}\right|}
$$

Equation (34) shows explicitly the general interrelations between each generalized dissipative conductivity $L_{i j}$ and the codispersion $\left\langle\Delta X_{i} \Delta X_{j}\right\rangle$ of observables $X_{i}$ and $X_{j}$.

In general, the dispersion-dissipation relations (34) involve the codispersions of all pair of observables in the set $X_{i}, X_{j}, R_{1}, \ldots, R_{z}$. For example, for a constituent with no non-Hamiltonian generators of the motion, if the state is such that $\sqrt{\rho}$ and $\sqrt{\rho} H$ are linearly independent, then the dispersion-dissipation relations become

$$
L_{i j}=\frac{1}{\tau}\left(\left\langle\Delta X_{i} \Delta X_{j}\right\rangle-\frac{\left\langle\Delta X_{i} \Delta H\right\rangle\left\langle\Delta H \Delta X_{j}\right\rangle}{\left\langle\Delta H^{2}\right\rangle}\right)
$$

where $\left\langle\Delta H^{2}\right\rangle=\langle\Delta H \Delta H\rangle$ is the dispersion in energy.

The dispersion-dissipation relations may be greatly simplified by choosing judiciously the set of observables $\left\{X_{j}\right\}$ spanning $\mathscr{L}_{s}(\mathscr{H})$. For example, for a given state $\rho$, we may consider a set $\left\{X_{j}\right\}$ such that

(a) $X_{0}=I, X_{1}=H, X_{2}=N_{1}, \ldots, X_{n+1}=N_{n}$;

(b) $\left\langle\Delta X_{j} \Delta H\right\rangle=0,\left\langle\Delta X_{j} \Delta N_{1}\right\rangle=0, \ldots,\left\langle\Delta X_{j} \Delta N_{n}\right\rangle=0$ for each $j>n+1$;

(c) $\left\{X_{j}\right\}$ is a quorum, i.e., the $X_{j}^{\prime}$ s are linearly independent. 
For such a set of observables $\left\{X_{j}\right\}$, we find $L_{i j}=0$ for $i$ (or $\left.j\right) \leqslant n+1$, and $\left\langle\Delta X_{j} \Delta R_{k}\right\rangle=0$ for every $j>n+1$ and $i \geqslant 1$. Hence, for the special set $\left\{X_{j}\right\}$ just defined and the given state $\rho$, the dispersion-dissipation relations (34) become

$$
L_{i j}=\frac{1}{\tau}\left\langle\Delta X_{i} \Delta X_{j}\right\rangle
$$

\section{CONCLUSIONS}

Quantum thermodynamics is a nonstatistical generalization of quantum theory encompassing within a single conceptual structure mechanics, equilibrium thermodynamics, and general nonequilibrium, including a fundamental deterministic description of irreversible processes.

In Ref. 1, we concluded that the nonlinear equation of motion adopted as the dynamical principle in quantum themodynamics is consistent with both the laws of mechanics and the laws of thermodynamics.

Here, we proved that the equation of motion is also consistent with the phenomenological theory of irreversible processes based on Onsager's reciprocity relations and the dispersion-dissipation relations. Within quantum thermodynamics, such relations emerge rigorously as exact theorems of the microscopic internal dynamics of each individual constituent of matter. The generalization of the present results to the general composite systems treated in Ref. 2 will be presented elsewhere.

\section{ACKNOWLEDGMENTS}

The author is indebted to Professor E. P. Gyftopoulos and Dr. G. N. Hatsopoulos, M.I.T., for helpful and stimulating conversations, and to Professor J. L. Park, Washington State University, for help in revising the manuscript. Financial support of the Thermo Electron Corporation is gratefully acknowledged.

\section{REFERENCES}

1. G. P. Beretta, Sc. D. Thesis, M.I.T. (1981), unpublished; G. P. Beretta, E. P. Gyftopoulos, J. L. Park, and G. N. Hatsopoulos, Nuovo Cimento B, 82, 169 (1984).

2. G. P. Beretta, E. P. Gyftopoulos, and J. L. Park, Nuovo Cimento B, 87, 77 (1985).

3. L. Onsager, Phys. Rev. 37, 405 (1931); 38, 2265 (1931).

4. H. B. G. Casimir, Rev. Mod. Phys. 17, 343 (1945). 
5. J. L. Park and R. F. Simmons, Jr., in Old and New Questions in Physics, Cosmology, Philosophy, and Theoretical Biology, A. van der Merwe, ed. (Plenum, New York, 1982).

6. H. B. Callen, Thesis, M.I.T. (1947), unpublished.

7. R. Kubo, J. Phys. Soc. Jpn. 12, $570(1957)$.

8. S. R. de Groot, Thermodynamies of Irreversible Processes (North-Holland, Amsterdam, 1951).

9. H. B. Callen, M. L. Barasch, and J. L. Jackson, Phys. Rev. 88, 1382 (1952).

10. L. Onsager and S. Machlup, Phys. Rev, 91, 1505 (1953).

11. S. R. De Groot, Thermodynamics of Irreversible Processes (Interscience, New York, 1950).

12. I. Prigogine, Introduction to Thermodynamics of Irreversible Processes (C. C. Thomas, Springfield, llinois, 1965).

13. R. D. Levine, J. Chem. Phys. 65, 3302 (1976).

14. G. N. Hatsopoulos and E. P. Gyftopoulos, Found. Phys. 6, 15, 127, 439, 561 (1976).

15. G. P. Beretta, Int. J. Theor. Phys. 24, 119, 1249 (1985).

16. G. P. Beretta, in Frontiers of Nonequilibrium Statistical Physics, G. T. Moore and M. O. Scully, eds. (Plenum, New York, 1985).

17. H. B. Callen and T. A. Welton, Phys. Rev. 83, 34 (1951); R. F. Greene and H. B. Callen, Phys. Rev. 83, 1231 (1951); H. B. Callen and R. F. Greene, Phys. Rev. 86, 702 (1952); R. F. Greene and H. B. Callen, Phys. Rev. 88, 1387 (1952).

18. H. Nyquist, Phys. Reo. 32, 110 (1928).

19. G. P. Beretta. J. Math. Phys. 27, 305 (1985).

20. W. Band and J. L. Park, Found. Phys. 1, 133 (1970). 